\title{
LÓGICA DA PESQUISA QUALITATIVA E QUESTÕES ÉTICAS: SÍNTESE INTEGRADORA E DESDOBRAMENTOS DOS PAINÉIS DO VI SIPEQ
}

\author{
LOGIC OF THE QUALITATIVE RESEARCH AND ETHICAL ISSUES: \\ INTEGRATING SYNTHESIS AND DEVELOPMENTS OF VI SIPEQ PANELS
}

\author{
Tiago Emanuel Klüber ${ }^{1}$
}

\begin{abstract}
Resumo: Expomos, neste texto, uma síntese integradora sobre os principais aspectos abordados nos três painéis do VI Seminário Internacional sobre Pesquisa e Estudos Qualitativos, SIPEQ, realizado em setembro de 2021, online. Destaca-se que a síntese decorre da fala de 15 pesquisadores renomados, de diferentes áreas do conhecimento que assumem a pesquisa qualitativa em suas práticas de pesquisa. Os aspectos foram registrados durante os painéis, com o auxílio de uma ferramenta online para a construção de "mapas mentais". Com essa ferramenta, além de pontuá-los, foi possível articulá-los, buscando convergências de sentido entre as falas. De modo geral, há aspectos filosóficos, teórico-epistemológicos, legais, operacionais e experienciais que foram aprofundados pelos painelistas. Dessas reflexões emergiram sugestões de encaminhamento que, conforme compreendemos, podem ser explicitadas em assertivas, como: a pesquisa qualitativa não se restringe às Ciências Humanas; os Comitês de Ética devem levar a cabo a composição equitativa das áreas; a inspiração em conselhos de ética internacionais, podem lançar mais luzes às condutas da Comissão Nacional de Ética em Pesquisa em Seres Humanos, CONEP e dos Comitês de Ética locais.
\end{abstract}

Palavras-chave: Ética em Pesquisa; Pesquisa Qualitativa; Procedimentos Éticos.

\begin{abstract}
In this text, we present an integrative summary of the main aspects addressed in the three panels of the VI International Seminar on Research and Qualitative Studies, SIPEQ, held in September 2021, online. It is noteworthy that the synthesis stems from the speech of 15 renowned researchers from different areas of knowledge who take qualitative research in their research practices. The aspects were registered during the panels, with the help of an online tool for the construction of "mental maps". With this tool, in addition to scoring them, it was possible to articulate them, seeking convergence of meaning between the lines. In general, there are philosophical, theoretical-epistemological, legal, operational and experiential aspects that were deepened by the panelists. From these reflections, suggestions for referral emerged that, as we understand, can be explained in assertions, such as: qualitative research not restricted to Human Sciences; the Ethics Committees must carry out the equitable composition of the areas; inspiration from international ethics councils, can shed more light on the conduct of the National Commission on Ethics in Research on Human Beings, CONEP and the local Ethics Committees.
\end{abstract}

Keywords: Research Ethics; Qualitative research; Ethical Procedures.

\section{Introdução}

O Seminário Internacional de Pesquisa e Estudos Qualitativos, SIPEQ, promovido pela Sociedade Brasileira de Estudos e Pesquisa Qualitativos, SE\&PQ, desde a sua primeira edição, realizada no ano de 2001, vem contribuindo para pensar o lócus da

\footnotetext{
${ }^{1}$ Doutor em Educação Científica e Tecnológica pela Universidade Federal de Santa Catarina, (UFSC). Professor Permanente do Programa de Pós-Graduação em Educação em Ciências e Educação Matemática (PPGECEM) na Universidade Estadual do Oeste do Paraná (UNIOESTE), Cascavel, PR, Brasil. E-mail: tiagokluber@gmail.com
} 
pesquisa qualitativa em diferentes áreas do conhecimento, como a Matemática, a Física, a Psicologia, a Sociologia, a Educação, a Biologia, a Química, entre outras, como se pode aferir nos textos publicados nos anais do evento ou na grade de programação editados ao longo de duas décadas ${ }^{2}$.

Nessa mesma direção, a sexta edição desse evento, buscou aprofundar um tema que se tornou cada vez mais presente na rotina dos pesquisadores brasileiros que realizam pesquisas que envolvem seres humanos, incidindo na necessidade da instauração de Comitês de Ética - CEP, nas diferentes instituições que realizam pesquisa com Seres Humanos e em regulamentações da Conselho Nacional de Saúde, CNS, sobre a composição da Comissão Nacional de Ética em Pesquisa em Seres Humanos, CONEP (RESOLUÇÃO, 446/2011).

A partir das exigências estabelecidas pelo Conselho, pela Resolução CNS 196/96, que estabeleceu diretrizes e normas regulamentadoras de pesquisas envolvendo seres humanos e por meio da Resolução 446/2011, toda e qualquer pesquisa envolvendo seres humanos passou a ser submetida ao crivo dos CEP. Desde então, pesquisadores experientes, novos ou em formação inicial que atuam nas mais diversas áreas do conhecimento têm por obrigação encaminhar os seus projetos, antes do início da pesquisa, para que sejam aprovados, do ponto de vista das questões éticas. Apenas com essa aprovação as pesquisas podem ser iniciadas. Em suma, tornou-se um regulador que se sobrepõe à expertise dos pesquisadores e também às diferentes abordagens de pesquisa que compreendem os aspectos éticos como inerentes à pesquisa e não apenas como protocolos de segurança e controle que são muito importantes para determinados tipos de pesquisa, mas não se aplicam a outros, principalmente àqueles que visam a compreensão situada e os aspectos humanos e sociais.

Essa obrigatoriedade trouxe uma nova rotina para os cursos de Graduação, Especializações lato sensu, Programas de Pós-Graduação stricto sensu, bem como para grupos de pesquisa. Desde o início, a Plataforma Brasil ${ }^{3}$, sistema que operacionaliza a submissão de todos os projetos, vem sofrendo críticas por pesquisadores de diferentes abordagens, principalmente das qualitativas. Estas entram em conflito com os muitos detalhes dessa Plataforma, que atendem ao modo de proceder de pesquisas quantitativas e biomédicas, evidenciando incomensurabilidade entre os modos de conceber e desenvolver pesquisas que não se alinham a este paradigma (KUHN, 1978).

\footnotetext{
${ }^{2}$ A programação pode ser encontrada em https://www.sepq.org.br/eventos_realizados.

${ }^{3}$ http://conselho.saude.gov.br/plataforma-brasil-conep?view=default
} 
Diante deste contexto e da crescente adesão de pesquisadores, principalmente de áreas que há duas ou três décadas não possuíam tradição acentuada de desenvolver pesquisa qualitativa, como a Administração, a Economia, a própria pesquisa em Saúde, a comissão organizadora do evento assumiu a tarefa de debater de maneira aprofundada e sob diferentes perspectivas, “[...] a lógica da Pesquisa Qualitativa e as solicitações da Plataformas Brasil”.

Esse tema foi aprofundado por meio dos três painéis abaixo mencionados, com as respectivas denominações e painelistas convidados:

- Lógica da Pesquisa Qualitativa e a questão da avaliação da pesquisa por comitês de ética: Dr. Luiz Gonçalves Junior - UFsCar - Brasil; Dra. Angela Ales Bello - Instituto de Fenomenologia, Roma, Itália; Dr. Egberto Ribeiro Turato Unicamp, SP; Dra. Maria Aparecida Viggiani Bicudo. UNESP, Rio Claro. São Paulo, Brasil; Dra. Maria Cecilia de Souza Minayo - Fundação Oswaldo Cruz (FIOCRUZ).

- Dificuldades encontradas pelos pesquisadores que fazem pesquisa qualitativa para submeterem suas propostas à Plataforma Brasil: Dra. Daniela Gomes dos Santos Biscarde - UFBA; Dra. Ettiène Guérios - UFPPR; Dra. Marília Josefina Marino - PUC-SP; Dra. Ana Maria Lopez Calvo de Feijoo, UFRJ; Dra. Luciana Puchalski Kalinke - UFPR;

- A plataforma Brasil: sua estrutura, sua lógica e aberturas para acolher pesquisas das Ciências Humanas: Dra. Diana Vidal - USP; Dr. Dartel Ferrari de Lima - Unioeste; Dra. Stella Regina Taquette - UERJ; Dr. José Artur Silva Emim - UNICSUL

As áreas de pesquisa dos referidos convidados são: Filosofia, Medicina, Enfermagem, Educação/Ensino, Matemática, Fisioterapia, Farmácia; Psicologia Clínica; Pedagogia; Sociologia/Saúde Pública; Ciências da Saúde/Engenharia Biomédica. Essa pluralidade de áreas de formação e atuação antecipa um argumento que foi fortemente debatido ao longo do evento: a pesquisa qualitativa não é exclusiva das Ciências Humanas, portanto, não se restringe a ela e, direta ou indiretamente, pode estar presente em nas Ciências em geral. Contribui, também, para fortalecer os argumentos dos aspectos apresentados na próxima seção. 


\section{Sobre os procedimentos para o destaque das ideias fortes dos painéis}

Ao longo das mais aproximadamente 10 horas de exposição e debates dos painéis, por certo, vários aspectos emergiram mais de uma vez, portanto, são convergentes. A ferramenta utilizada para registrar, manusear as anotações e mapear as ideias foi o mindmeister ${ }^{4}$. Na figura 1, apresentamos um exemplo das ideias fortes que se destacaram no primeiro painel. Analogamente, procedeu-se do mesmo modo para os demais painéis individualmente e entre eles, para a exposição do painel IV: Análise do apresentado e debatido nas Mesas redondas e sugestões de encaminhamento de propostas para a Plataforma Brasil. Coordenação: Dr. Tiago Emannuel Klüber - Unioeste; Dr. Marcelo de Carvalho Borba - UNESP, Rio Claro; Dra. Maria Ines Fini - ANEBHI. O registro sistemático e a manipulação dos mapas favoreceu a construção concisa deste texto e uma memória mais precisa.

Figura 1: Ideais fortes do Painel I: Lógica da Pesquisa Qualitativa

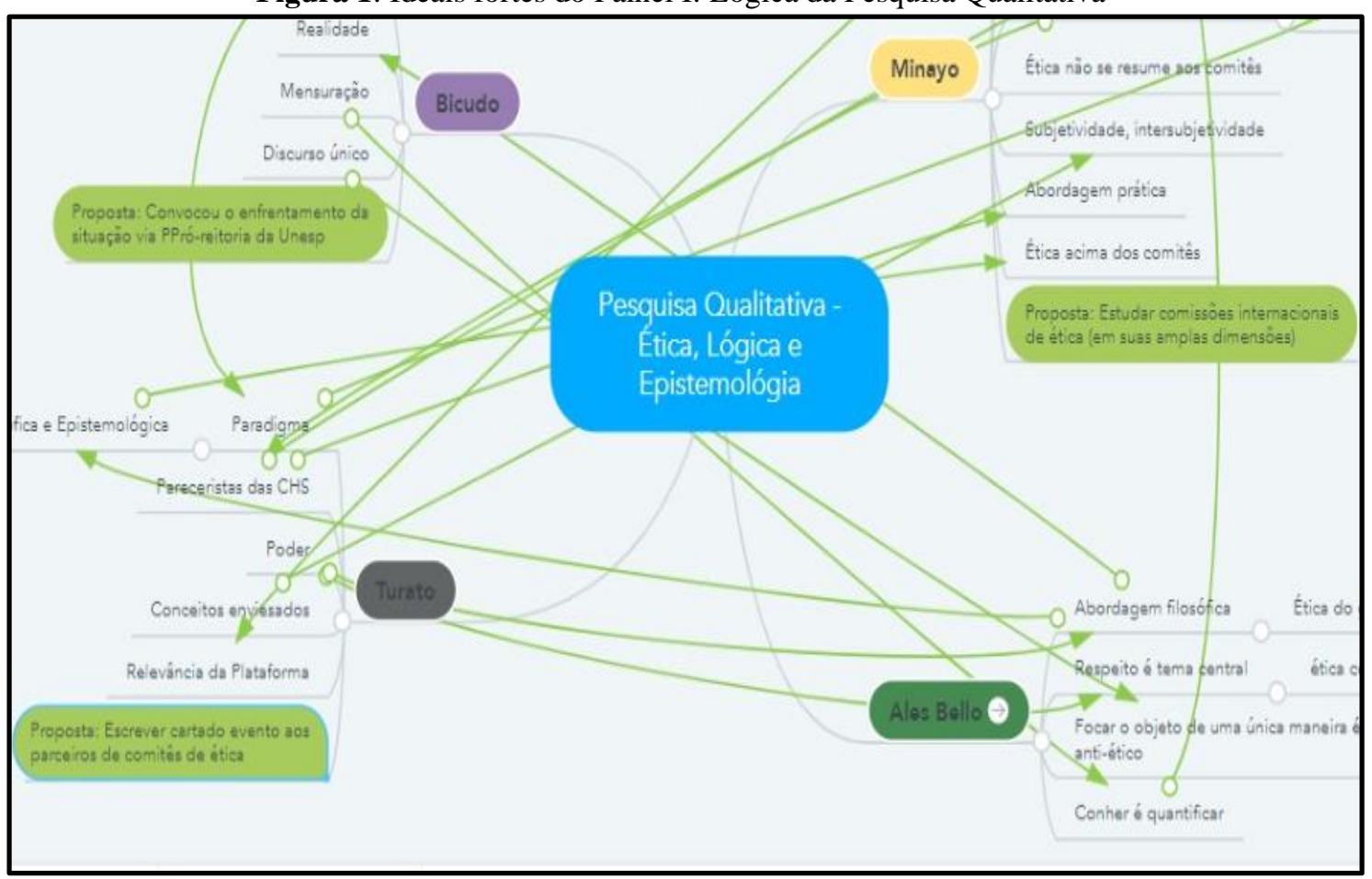

Fonte: Acervo próprio (2021)

Esse movimento de registro, acompanhado da leitura dos resumos dos textos submetidos pelos autores e consulta aos textos enviados pelos painelistas, bem como da reflexão demorada sobre as ideias fortes abordadas nos painéis, permitiram chegar à síntese que ora expomos.

\footnotetext{
${ }^{4}$ Disponível em: www.mindmeister.com, na versão gratuita, online. Há diferentes versões para o software.
} 


\section{Os aspectos destacados nos painéis}

O modo pelo qual elaboramos essa síntese, como se pode depreender do exemplo da figura, não é linear, assim como o nosso pensamento não é linear, ainda que possa ser organizado e explicitado com sentido de linearidade. Assim, os aspectos aqui apresentados são resultado do movimento de idas e vindas sobre cada uma das falas e do nosso olhar atento aos pontos de confluência e também do diálogo entre os coautores deste texto.

Em primeiro lugar, por questão de organização do itinerário textual, trazemos traços dos aspectos filosóficos concernentes à lógica da pesquisa qualitativa e à dimensão ética. Em todos os painéis, em maior ou menor grau de aprofundamento, estes aspectos foram mencionados. Eles tocam temas centrais da filosofia como, respeito, costumes $e$ valores, poder, realidade, critérios de conhecimento (quantificar, mensurar, visão monolítica); o problema da apropriação histórica para os pesquisadores em diferentes campos; o ser é inseparável do conhecer;

Imediatamente, articuladas às questões filosóficas, se encontram as questões teórico-epistemológicas, que envolvem as noções de subjetividade, intersubjetividade, paradigma; história do conhecimento, modos de proceder e a instrumentação da pesquisa qualitativa e não qualitativa; abordagens de pesquisa para além das experimentais, mais especificamente das áreas biomédicas e farmacêuticas, a vida como valor. A ideia implícita nesta afirmação é a de que a vida não pode estar a serviço do progresso da ciência, como ocorreu em períodos críticos para a história da humanidade, como foi o da II Guerra Mundial. O conhecimento das atrocidades realizadas em nome da ciência gerou um debate amplo a respeito de a Ética, em sentido positivo, não poder se separar do fazer científico.

Os aspectos legais foram amplamente discutidos, recorrendo à história da criação de Comitês de Ética internacionais, aos marcos históricos e à necessária implementação dos Conselhos e Comitês de Ética em pesquisa mundo afora e também no Brasil. No Brasil, o Conselho Nacional de Saúde instaurou a CONEP em 1996. As atividades que desenvolveu culminaram em um instrumento de avaliação que permitiu maior agilidade, abrangência e explicitação de critérios, o que conduziu à criação e implantação da Plataforma Brasil, que se tornou um órgão regulador e de controle e, também, em um repositório de pesquisas desenvolvidas em todo país. Esta última característica foi considerada, entre painelistas, um aspecto altamente positivo e que indica a pertinência 
da Plataforma, dada a disponibilização das pesquisas de todo território nacional, que estão em andamento.

Desde a criação do Conselho Nacional sobre ética em Pesquisa Seres Humanos, CONEP, tem havido mudanças nos seus instrumentos legais, como as trazidas nas Resoluções 466/2012, 510/2016 do CNS. Evidenciou-se, claramente nas falas dos debatedores, que há avanços no acolhimento das pesquisas qualitativas, principalmente, pela aceitação de características e modos próprios de as Ciências Humanas procederem. Foi apontado que há, ainda, desconhecimento e descompasso do expresso na legislação e as práticas do Conselho e dos Comitês institucionais locais. Um dos principais aspectos destacados é a não observância de os colegiados serem equânimes em relação às áreas do conhecimento.

No tocante aos aspectos operacionais, destacou-se que eles decorrem tanto dos aspectos filosóficos como teórico-epistemológicos, uma vez que a plataforma foi concebida segundo uma lógica exclusivista, ou seja, aquela implícita à ciência positivista, não dando conta da pluralidade dos outros modos de se fazer pesquisa que não sejam quantitativas e experimentais. Sendo assim, há clara divergência terminológica, o que muitas vezes inviabiliza o início das pesquisas, trazendo prejuízos éticos aos pesquisadores, sob o argumento de proteção apenas aos sujeitos participantes, quando as questões éticas deveriam salvaguardas a ambos. A formação dos integrantes dos membros de Comitês de Ética, em geral, está restrita às suas áreas de atuação, muitas vezes sem conhecimentos mínimos de Epistemologia, Filosofia da Ciência e Filosofia, incidindo em compreensões enviesadas acerca do mérito pesquisa, portanto, sem clara compreensão do conteúdo, do "o quê" deve ser avaliado e, portanto, do modo de avaliar. Outro aspecto operacional relevante é a questão do anonimato das pesquisas. Segundo foi destacado por Taquete, em alguns casos, como na História Oral, o anomimato que se apresenta como um protocolo de proteção pode se tornar um ato antiético e de violência para com os participantes que por princípio devem ser identificados. Portanto, a questão é deixar aberta a possibilidade aplicação ou não do anonimato às pesquisas.

Os aspectos experienciais evidenciam as dificuldades de membros e presidentes dos Comitês de Ética Locais, mas também revelam que há experiências salutares, no âmbito dos comitês. Há conflitos, muitas vezes, beirando o irracional, também por parte dos pesquisadores que não aceitam avaliações dos comitês, assim como, entre os próprios avaliadores que abrem pouco espaço para compreender a perspectiva dos colegas. 
Esses aspectos aqui pontuados conduziram a um diálogo equilibrado, evidenciando a importância da implantação, consolidação e extensão dos Comitês de Ética, porém, indicando que há se estabelecer um diálogo mais intenso e mesmo regulamentado, principalmente dos pesquisadores qualitativos, participando e contribuindo na construção e estabelecimento de diretrizes claras que acolham pesquisas qualitativas de todas as áreas. Assim, pode-se desmistificar tanto a dicotomia quantitativo x qualitativo, bem como a ideia de que a ciência se reduza apenas ao mensurável. Entendeu-se que a pesquisa qualitativa não é um modo de proceder exclusiva das Humanas e Sociais, ainda que tenha se consolidado nestas tradições. Ela é inerente ao conhecimento, de um modo ou de outro, sempre se reporta à qualidade.

\section{Encaminhamentos depreendidos dos Painéis}

Esta seção contém as sugestões de encaminhamentos que foram apontados pelos painelistas, assim como as nossas compreensões concernentes a elas.

A Dra. Angela Ales Bello recomendou que se abra uma reflexão ampla sobre as questões originárias do sentido de ética em pesquisa, de tal maneira que se compreenda que os objetos, mesmo podendo ser focados em alguma dimensão específica, não podem perder o sentido do todo.

A Dra. Maria Cecilia Minayo sugeriu perseguir aquilo é preconizado na Resolução 510/2016 do CNS, buscando a composição equânime dos membros que integrarão os comitês, segundo as áreas e ainda se inspirar nos conselhos internacionais de ética em pesquisa, para compreender os procedimentos éticos, ampliando a experiência brasileira.

A Dra. Maria Aparecida Viggiani Bicudo convocou a Pró-Reitoria da Unesp para capitanear a apresentação de um documento sobre aquilo seria levantando durante o evento. Assim, foi entendido, pois os entraves sofridos por pesquisadores qualitativos é uma realidade muito presente tanto na UNESP, quanto em outras instituições.

O Dr. Egberto Ribeiro Turato indicou que há que se investir na formação Epistemológica dos membros do comitê, bem como defendeu que se faça uma carta aos parceiros dos comitês de ética, para congregar forças de um movimento de base que dê conta de propor essas questões ao CONEP. Apontou, também, a questão do poder que perpassa os Comitês de Ética, em uma dimensão micro, e em uma dimensão macro, envolvendo a CONEP. 
Os Doutores Ana Maria Feijoo, Dartel Ferrari de Lima e Stella Regina Taquette convergiram para a necessidade de investir em uma maior interação entre membros dos comitês e pesquisadores, de maneira a sanar dúvidas pontuais, bem como, permitir uma compreensão mais ampla dos diferentes modos de produzir pesquisa qualitativa.

O Dr. Tiago Emanuel Klüber sugeriu que, a partir destes aspectos supramencionados, seja elaborado um documento com diretrizes mínimas para a composição dos comitês, ou seja, que se elabore uma interpretação dos aspectos contidos na Resolução 510/2016, acerca do lugar da pesquisa qualitativa, sem reforçar a dualidade Ciências da Natureza/Ciências Humanas, qualitativa quantitativa, com bem expôs a Daniela Gomes dos Santos Biscarde.

A Dra. Maria Inês Fini sugeriu a construção de um "decálogo", com os princípios éticos a serem seguidos no âmbito dos comitês de ética.

O Dr. Marcelo de Carvalho Borba sugeriu a construção de uma grade de avaliação geral para pesquisas qualitativas para a aprovação "direta" de pesquisa semelhantes.

\section{Olhando para a síntese e pensando sobre desdobramentos}

A riqueza das exposições e a qualidade dos debates permitiram compreender que a dimensão da Ética precede os aspectos procedimentais e protocolares. Assumindo a dimensão do respeito, do pensar crítico, porém, aberto e dialógico, pode-se superar a negação da pesquisa qualitativa, pois uma visão monolítica sobre o modo de se dirigir aos fenômenos fere eticamente as condições de produção do conhecimento. Essa interpretação encontra convergência quando Bombassaro (1994) afirma que a uma solução para superar a dualidade entre a Racionalidade decorrente das Ciências da Natureza, aquela centrada em questões matemáticas e metodologicamente controladas e a Historicidade das Ciências Humanas e Sociais, que foca a práxis humana e os sentidos próprios que decorrem dos seres humanos, é aceitação do logos hermenêutico (STEIN, 2004). Este logos se refere às condições originárias para a produção do conhecimento, portanto, é no logos hermenêutico que o conhecimento se torna possível, é nele que o conhecimento produzido é tecido, constituído. É nesta região que toda lógica estruturada, matemática e ciências em geral são gestadas. Portanto, ao contrário do que sempre se pensou, que a interpretação é um lugar decaído, de menor importância para as ciências, isso se modifica ao aceitar a dimensão hermenêutica, na qual o qualitativo pode atingir o seu ápice, como compreensão enquanto um modo de ser. 
Nesse sentido, parece haver em curso, um caminho que, ainda que lentamente perfilado, passou a reconhecer e compreender a relevância das Ciências Humanas e Sociais, como já disse, mais de uma vez, por meio da Resolução 510/2016, CNS. Mais importante do que o acolhimento das particularidades das Ciências Humanas e Sociais em uma resolução, é a compreensão de que o qualitativo não se restringe a estas áreas, mas perpassa as diferentes áreas, ainda que não seja amplamente aceito deste modo.

Esse entendimento é convergente à ideia exposta por Bombassaro (1994), pois, dar ênfase às qualidades ou quantidades, sempre decorre da apropriação de discursos que permitem dar conta daquilo que se está estudando e não apenas tentar buscar o previamente dado.

Dizer que o qualitativo não é exclusividade das Ciências Humanas e Sociais também não quer dizer que toda pesquisa se resuma à pesquisa qualitativa enunciada, mas assumir a radicalidade que, de um modo de outro, sempre há a participação das qualidades, o "lado qualitativo", que longe de ser puramente subjetiva e, portanto, desprezível, é uma condição sine qua non. É nesse sentido que compreendemos com Husserl (1996) que matéria e qualidade não se separam no conhecimento, pois são momentos (partes não-independentes).

\section{Referências}

BOMBASSARO, L. C. As fronteiras da epistemologia: uma introdução ao problema da racionalidade e da historicidade do conhecimento. Rio de Janeiro: Vozes, 1992.

BRASIL. CONSELHO NACIONAL DE SAÚDE. Resolução CNS n 446/2011 define e caracteriza o funcionamento do conselho de ética em pesquisa no Brasil. Brasília: CNS, 2011.

BRASIL. CONSELHO NACIONAL DE SAÚDE. Resolução no 466/2012 trata de pesquisas e testes em seres humanos. Brasília: CNS, 2012.

BRASIL. CONSELHO NACIONAL DE SAÚDE. Resolução CNS n 510/2016 determina diretrizes éticas específicas para as ciências humanas e sociais. Brasília: CNS, 2016.

HUSSERL, E. Investigações lógicas: sexta investigação. São Paulo nova cultural, 1996, p. 523. (Os Pensadores).

KUHN, T. A estrutura das revoluções Científicas. Trad. Beatriz Vianna Boeira e Nelson Boeira. São Paulo: Perspectiva, 1978.

STEIN, E. Aproximações sobre hermenêutica. 2. ed. Porto Alegre: EDIPUCRS, 2004. (Coleção Filosofia). 